\title{
Application of Bayesian network in Production Line System Reliability Analysis
}

\author{
Zhai Sheng \\ Dalian Institute of Science and Technology, P.R. China \\ zhaishengdl@163.com \\ *Zhai Sheng
}

Keywords: Bayesian network, Multi-failure mode, Reliability analysis, Production line.

\begin{abstract}
Considering the multi-state and uncertainty of causality in complex systems, a method for reliability modeling and assessment of a multi-state system based on Bayesian Network (BN) is proposed by using the advantage of uncertainty reasoning and multi-state expression of BN. The proposed method is able to identify the system weakness through calculating the system reliability on the basis of multi-state probabilities of elements. The model is applied to a production line system to verify its effectiveness.
\end{abstract}

\section{Introduction}

With the rapid development of modern industrial technology, the equipment systems are becoming more and more complex. Faced with increasingly complex systems, the requirements for system reliability analysis are getting stringent.

Cell production line is a complex system of mechanical, electrical and liquid coupling with complex structures and many components. The multi-states are presented in the system and components. The failure modes are diverse, dependent and uncertain, which make the application of the traditional reliability theory restricted a lot.

For this reason, Xue[1] and Liu[2] studied the application of fault tree method from the two-state system to the multi-state system. Wang et al. [3] analyzed the reliability of multi-state system by using fault tree. These methods broaden the range of traditional reliability analysis methods, but the fundamental problems are not solved.

In recent years, as an advanced technology of uncertainty knowledge representation and inference, the Bayesian network (BN) is widely applied to the reliability analysis [4], risk analysis[5], fault diagnosis [6] of the complex system, etc.

In this paper the BN model will be introduced to the reliability analysis of cell production line system. Combining with the inference mechanism of BN model, the bucket algorithm method is used to calculate the system reliability and the posteriori probability. Finally by analyzing the reliability of the cell production line system, the feasibility and practicability of the BN model in reliability analysis of complex system are verified.

\section{Bayesian Network (BN)}

A BN is a directed acyclic graph (DAG), in which the nodes represent variables, arcs signify direct causal relationships between the linked nodes, and the conditional probability table(CPT) assigned to the nodes specify how strongly the linked nodes influence each other[7]. A BN is defined by a set of nodes and a set of directed arcs. A probability is associated to each state of the node. This probability is defined, a priori for a root node and computed by inference for the others. The computation is based on the probabilities of the parent's states and the CPT.

Assuming that $x_{1}, x_{2}, \ldots, x_{l}$ are the nodes of $\mathrm{BN}$, the joint probability of all nodes in the network as follows: 


$$
P(X)=P\left(x_{1}, x_{2}, \cdots, x_{l}\right)=P\left(x_{1}\right) P\left(x_{2} \mid x_{1}\right) \cdots P\left(x_{l} \mid x_{1}, x_{2}, \cdots, x_{l-1}\right)=\prod_{i=1}^{l} P\left(x_{i} \mid x_{1}, x_{2}, \cdots, x_{i-1}\right)
$$

If $A\left(x_{i}\right)$ represents a subset of any node consisted of non-descendant nodes, indicating the direct parent node of $x_{i}$ with $\pi\left(x_{i}\right)$, then according to the conditional independence assumptions, there is:

$$
\begin{aligned}
& P\left(x_{i} \mid A\left(x_{i}\right), \pi\left(x_{i}\right)\right)=P\left(x_{i} \mid \pi\left(x_{i}\right)\right) \\
& P\left(x_{1}, x_{2}, \ldots, x_{l}\right)=\prod_{i=1}^{k} P\left(x_{i} \mid \pi\left(x_{i}\right)\right)
\end{aligned}
$$

\section{BN Model}

\subsection{BN Model Construction}

Because fault tree analysis plays a very important role in traditional analysis methods, fault tree modeling is usually used to establish BN model, that is to set up fault tree according to the logical relationship between variables, and then map the fault tree model into BN model.

Assuming that the system and the components have three states, which are represented by 0,1 , and 2. 0 state is the normal state, 1 state is the serious fault state, and 2 is the slight fault state.

The BN model of the basic series and parallel system is described below.

1) Series System

It is assumed that the system $T$ consists of two three-state components, $x_{1}$ and $x_{2}$ in series. $P$ represents the probability of a system or component. There are: when either $x_{1}$ or $x_{2}$ is in one serious fault state, the system $T$ is in the fault state; When $x_{1}$ and $x_{2}$ are both in slight fault states, the system $T$ is in a slight fault state; When $x_{1}$ and $x_{2}$ are normal, the system $T$ is normal.

The BN model of the series system is shown in figure 1(a).

The probability is calculated by using the bucket elimination method, as follows:

$$
\begin{aligned}
P & (T=1)=\sum_{T=1} P\left(x_{1}, x_{2}, T\right)=\sum_{T=1} P\left(T=1 \mid x_{1}, x_{2}\right) P\left(x_{1}, x_{2}\right)=\sum_{T=1} P\left(T=1 \mid x_{1}, x_{2}\right) P\left(x_{1}\right) P\left(x_{2}\right) \\
= & P\left(T=1 \mid x_{1}=0, x_{2}=1\right) P\left(x_{1}=0\right) P\left(x_{2}=1\right)+P\left(T=1 \mid x_{1}=1, x_{2}=0\right) P\left(x_{1}=1\right) P\left(x_{2}=0\right) \\
& +P\left(T=1 \mid x_{1}=1, x_{2}=1\right) P\left(x_{1}=1\right) P\left(x_{2}=1\right)+P\left(T=1 \mid x_{1}=1, x_{2}=2\right) P\left(x_{1}=1\right) P\left(x_{2}=2\right) \\
& +P\left(T=1 \mid x_{1}=2, x_{2}=1\right) P\left(x_{1}=2\right) P\left(x_{2}=1\right)
\end{aligned}
$$

In this way, according to the prior probability and CPT, the probability $P(T=1)$ of the serious fault state of the series system can be calculated.

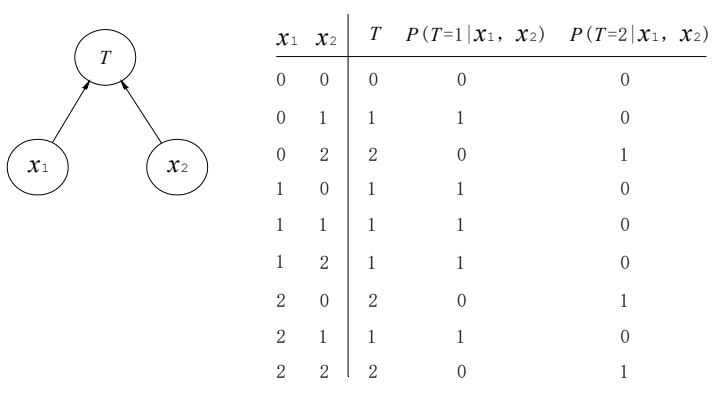

(a)

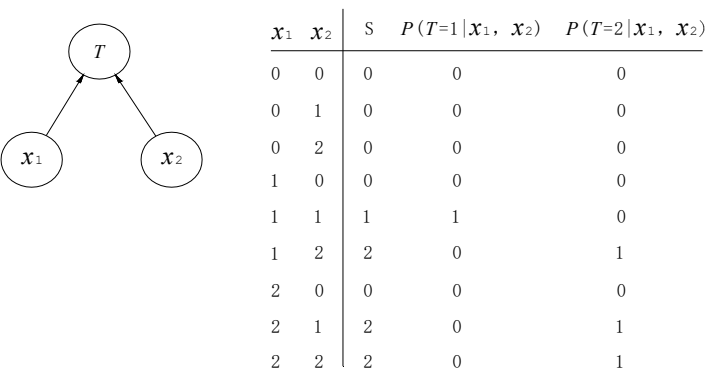

(b)

FIG. $1 \mathrm{BN}$ model of the series and parallel system

\section{2) Parallel System}

It is assumed that the system $T$ consists of two three-state components, $x_{1}$ and $x_{2}$ in parallel. $P$ represents the probability of a system or component. There are: when either $x_{1}$ or $x_{2}$ is in a slight fault state, the system $T$ is in a slight fault state; When $x_{1}$ and $x_{2}$ are both in the serious fault states, the system $T$ is in the serious fault state; When $x_{1}$ and $x_{2}$ are normal, the system $T$ is normal.

The BN model of the parallel system is shown in figure 1(b).

The probability is calculated by using the bucket elimination method as follows:

$$
\begin{aligned}
P(T=1) & =\sum_{T=1} P\left(x_{1}, x_{2}, T\right)=\sum_{T=1} P\left(T=1 \mid x_{1}, x_{2}\right) P\left(x_{1}, x_{2}\right)=\sum_{T=1} P\left(T=1 \mid x_{1}, x_{2}\right) P\left(x_{1}\right) P\left(x_{2}\right) \\
& =P\left(T=1 \mid x_{1}=1, x_{2}=1\right) P\left(x_{1}=1\right) P\left(x_{2}=1\right)
\end{aligned}
$$


Similarly, according to the prior probability and CPT, the probability $P(T=1)$ of the serious fault state of the parallel system can be calculated. The probability of $P\left(T=1 \mid x_{2}=1\right)$ and $P\left(x_{2}=1 \mid T=1\right)$ can also be worked out.

\subsection{Reliability Analysis Based on BN Model}

1) Prediction of System Reliability.

Before the system failure occurs, by taking advantage of the prior probability and CPT of the known nodes, through $\mathrm{BN}$ inference algorithm, the system fault probability can be obtained. This is the prediction, also known as causal inference. The calculation of $P(T=1)$ is a kind of causal inference, and the result of $P(T=1)$ is the reliability of the system.

When a root node fails, it can be further inferred that influence degree of the node on the system, namely, $P\left(T=1 \mid x_{1}=1\right)$, and it is also causal inference. And, of course, $P\left(T=1 \mid x_{1}=2\right)$ can also be calculated. In this way, the influence degree of each node on the system can be analyzed.

2) Diagnosis of system failure.

When the system fault occurs, according to the BN model, by using the prior probability and CPT of the known node, through BN inference algorithm, the fault probability of each root node can be calculated, which is the posteriori probability. The fault posterior probability of the root node is arranged in order from big to small, and eventually the corresponding root node can be inferred. This is a process of diagnosis, or the diagnosis inference. In fact, that's the inverse inference of BN. The calculation of $P\left(x_{1}=1 \mid T=1\right)$ is a kind of diagnosis inference. By analogy, the probability of $P\left(x_{2}=1 \mid T=1\right), P\left(x_{2}=2 \mid T=1\right)$ can be found out and arranged in order, and finally the most likely event that leads to the system fault can be found out.

\section{Reliability analysis of cell production line system}

The reliability analysis data of the cell production line system of an enterprise are presented as follows.

Table 1 Event list

\begin{tabular}{cc||cc}
\hline Symbol & Event & Symbol & Event \\
\hline$T$ & Subsystem feeding $\mathrm{MnO}_{2}$ fault & $x_{5}$ & Sensor fault \\
$M_{1}$ & Device feeding negative shell fault & $x_{6}$ & Defective $\mathrm{MnO}_{2}$ \\
$M_{2}$ & Device feeding positive $\mathrm{MnO}_{2}$ fault & $x_{7}$ & Cylinder feeding $\mathrm{MnO}_{2}$ jam \\
$M_{3}$ & Out-feeding device fault & $x_{8}$ & Solenoid valve fault \\
$M_{4}$ & Cylinder feeding negative shell fault & $x_{9}$ & Conveyor belt fault \\
$M_{5}$ & Cylinder feeding $\mathrm{MnO}_{2}$ fault & $x_{10}$ & Sensor fault \\
$x_{1}$ & Insufficient air pressure & $x_{11}$ & Insufficient air pressure \\
$x_{2}$ & Cylinder feeding negative shell jam & $x_{12}$ & Out-feeding cylinder jam \\
$x_{3}$ & Solenoid valve fault & $x_{13}$ & Sensor fault \\
$x_{4}$ & Conveyor belt fault & & \\
\hline
\end{tabular}

The system is divided into five working subsystems, namely the subsystem feeding negative shell and lithium, subsystem dividing the paper into negative shell, subsystem feeding $\mathrm{MnO}_{2}$, subsystem assembling positive steel shell, sealing subsystem. Among them the subsystem feeding $\mathrm{MnO}_{2}$ is selected for analysis.

\subsection{BN reliability modeling}

According to the logical relationship between events, the fault tree model which top event is subsystem fault feeding $\mathrm{MnO}_{2}$ is established, Specification between the events and the notations is shown in table 1.

According to the fault tree model, it is mapped to the BN model as shown in figure 3 . 


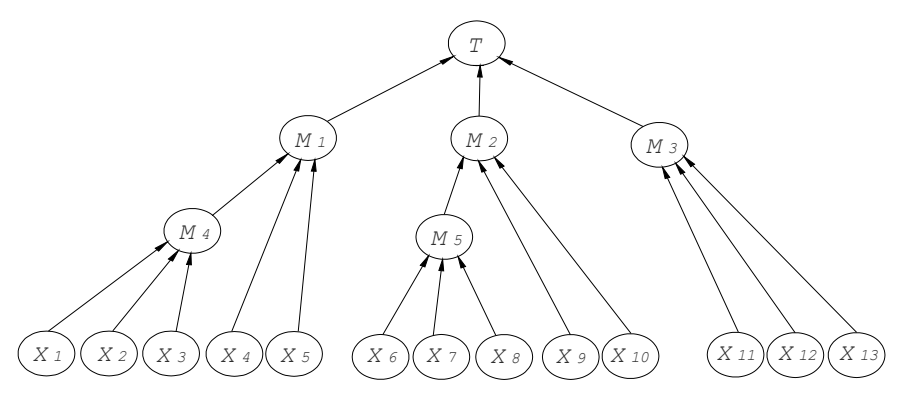

FIG. $3 \mathrm{BN}$ model of subsystem feeding $\mathrm{MnO}_{2}$

\section{2 $\mathrm{BN}$ reliability analysis}

The failure rate data of root node is provided by an enterprise, and the prior probability of the occurrence of each event is shown in table 2:

Table 2 priori probability table of nodes $\left(10^{-5} / \mathrm{h}\right)$

\begin{tabular}{cc||cc}
\hline node & $x_{i}=1, x_{i}=2$ & node & $x_{i}=1, x_{i}=2$ \\
\hline$x_{1}$ & $0,0.042$ & $x_{8}$ & $0.011,0.023$ \\
$x_{2}$ & $0,0.325$ & $x_{9}$ & $0.032,0$ \\
$x_{3}$ & $0.012,0.024$ & $x_{10}$ & $0.016,0.027$ \\
$x_{4}$ & $0.026,0$ & $x_{11}$ & $0,0.053$ \\
$x_{5}$ & $0.014,0.026$ & $x_{12}$ & $0,0.075$ \\
$x_{6}$ & $0,0.033$ & $x_{13}$ & $0.013,0.021$ \\
$x_{7}$ & $0,0.167$ & & \\
\hline
\end{tabular}

For $x_{1}, x_{2}$, etc., are irrelevant to class $\mathrm{A}$ failure modes, and $\mathrm{x}_{4}$ and $\mathrm{x} 9$ are not related to class $\mathrm{B}$ fault modes, they are binary states namely $P\left(x_{1}=1 \mid T=1\right)=0, P\left(x_{4}=2 \mid T=2\right)=0$. Multiple states can coexist in $\mathrm{BN}$, which greatly improves the flexibility of modeling.

1) Prediction. According to the prior probability and CPT of the node, the reliability or failure rate of the leaf node and intermediate node is calculated when the system is not in fault state.

In $\mathrm{BN}$ model of subsystem feeding $\mathrm{MnO}_{2}$, according to the prior probability of $x_{1}$ to $x_{13}$, intermediate nodes and $T$ 's CPT, by using the bucket elimination method to calculate the probability, and the probability of the top event $T$ is inferred to be $P(T=1)=0.124 \%, P(T=2)=0.812 \%$, and the probability of nodes $M_{1}, M_{2}$, and $M_{3}$ is calculated. In this way, the probability of node $T, M_{1}, M_{2}$ and $M_{3}$ can be predicted in the case of known node prior probability, so that the reliability of each link in the system can be understood when no fault occurs.

2) Diagnosis. According to the prior probability of the node and CPT, the reliability or failure rate of the root node and intermediate nodes is calculated when the system failure occurs, and the reliability or failure rate of the leaf nodes and intermediate nodes is calculated when the components failure occurs.

In the state of the system $T=1$, the posterior probability of each node is shown in table 3 .

In table 3, the $\mathrm{x}_{1}, \mathrm{x}_{2}$, etc. are irrelevant to class A fault (state 1), that is $P\left(x_{1}=1 \mid T=1\right)=0$,

Table 3 at $T=1$, the posterior probability of each node $P\left(x_{\mathrm{i}} \mid T=1\right)\left(10^{-5} / \mathrm{h}\right)$

\begin{tabular}{cc||cc}
\hline node & $x_{i}=1, x_{i}=2$ & node & $x_{i}=1, x_{i}=2$ \\
\hline$x_{1}$ & $0,0.042$ & $x_{8}$ & $8.876,0.021$ \\
$x_{2}$ & $0,0.325$ & $x_{9}$ & $25.820,0$ \\
$x_{3}$ & $9.682,0.022$ & $x_{10}$ & $12.910,0.024$ \\
$x_{4}$ & $20.979,0$ & $x_{11}$ & $0,0.053$ \\
$x_{5}$ & $11.296,0.023$ & $x_{12}$ & $0,0.075$ \\
$x_{6}$ & $0,0.033$ & $x_{13}$ & $10.489,0.019$ \\
$x_{7}$ & $0,0.167$ & & \\
\hline
\end{tabular}


$P\left(x_{2}=1 \mid T=1\right)=0$, and when class A failure occurs, they may and may not appear, so the posteriori probability $P\left(x_{1}=2 \mid T=1\right), P\left(x_{2}=2 \mid T=1\right)$ does not change. According to table 3 , when the system is in state 1 , the most influential factor is $x_{9}$.

In the state of the system $T=2$, the posterior probability of each node is calculated, and the most influential factor is $x_{2}$.

To sum up, since the state 1 is a heavy fault state, which has a large impact on the system, so the problem of $x 9$ is of particular concern.

In this case, the probability $P\left(x_{1} \mid T=1\right)$ and $P\left(x_{1} \mid T=2\right)$ of other nodes can be obtained when the system failure mode ( $T=1$ or $T=2)$ is known. In turn, the conditional probability of $x_{1}$ up to $x_{13}$ can be calculated. Therefore, the key problem is the conveyor belt fault of the device feeding positive $\mathrm{MnO}_{2}$, which has the greatest influence on the system.

\section{Conclusion}

BN has a flexible modeling framework in terms of the reliability analysis of multi-state systems. It has such characteristics as the rigorous mathematical formula derivation, uncertainty and expression ability of multi-state events. It can directly and clearly express the influence relationship between multi-state systems and components, through bidirectional reasoning to identify the weak links of the system. The proposed method solves the limitation of traditional reliability theory better, and the system reliability analysis can be realized more comprehensively. For this purpose, the reliability analysis and modeling method based on BN multi-state system is applied in the cell production line system, and it provides reference for the reliability analysis of the complex system in the future.

\section{References}

[1] J. Xue. On Multistate System Analysis, IEEE Transactions on Reliability, vol.34, pp. 329-337, 2009.

[2] C. Liu, N. Chen, and J. Yang, Reliability analysis of servo turret based on multi-state fault tree, Journal of Southeast University, vol.44, pp. 538-543, 2014.

[3] X. M. Wang, L. Y. Xie, and J. Y. Zhou, Reliability model of fractional ordered system, Chinese Journal of Mechanical design, vol.21, pp. 6-9, 2004.

[4] P. Baraldi, L. Podofillini, L. Mkrtchyan, et al, Comparing the treatment of uncertainty in Bayesian networks and fuzzy expert systems used for a human reliability analysis application. Reliability Engineering \& System Safety, vol.138, pp. 176-193, 2015.

[5] M. Yazdi, S. Kabir, A Fuzzy Bayesian Network approach for Risk Analysis in Process Industries. Process Safety \& Environmental Protection, vol. 111, pp. 507-519, 2017.

[6] L. J. M. Aslett, F. P. A. Coolen, and S. P. Wilson, Bayesian Inference for Reliability of Systems and Networks Using the Survival Signature, Risk Analysis, vol.35,pp. 1640-1651, 2015.

[7] H. Langseth, L. Portinale, Bayesian networks in reliability, Reliability Engineering System Safety, vol.92, pp.92-108, 2007. 